\title{
University-Industry Programs as Platforms: A Case Study of Multi- Disciplinary Collaborative Network Development
}

\author{
Arash Hajikhani \\ Lappeenranta University of Technology \\ arash.hajikhani@lut.fi \\ Jason Wilmot \\ Stanford University \\ jwilmot@stanford.edu
}

\author{
Karina Alexanyan \\ Stanford University \\ karinaa@stanford.edu
}

\author{
Erin Young \\ University of Oxford \\ erin.young@education.ox.ac.uk
}

\author{
Martha G. Russell \\ Stanford University \\ marthar@stanford.edu
}

\begin{abstract}
This paper shows how University-Industry (UI) liaisons can be studied in light of the emerging and insightful literature on platforms. Applying the concept of platforms to University-Industry programs, this paper describes one industry affiliate program and analyzes its synergistic impact on multidisciplinary involvement and collaboration network development. Insights and recommendations are made for catalyzing mutually beneficial collaborations through research themes as mechanisms for platform orchestration.
\end{abstract}

\section{Introduction}

Researchers describe companies such as Facebook, Google and Apple as Industry Platforms [1] and scientific discourse about platforms is rapidly expanding into other industries, such as banking, healthcare, energy and transportation [2]-[5]. As platforms bring together multiple user groups, they create network effects or network externalities [1]. The greater the number of users who adopt the platform, the more valuable the platform becomes to the owner and to the users because of growing access to the network of users and often to a growing set of complementary innovations [6].

On the other hand, the importance of intermediate structures such as University-Industry programs for effective collaboration and knowledge exchange has been widely acknowledged by academics and practitioners [7]. UI as Intermediaries are commonly understood to be bridging organizations, which help to develop bilateral or multilateral relationships [8]. Opportunities to develop insights with strategic value increase with interdisciplinary and multidisciplinary research [9]. In the history of academic knowledge generation, interaction with industry has been suggested as a mechanism to inspire research and reveal business applications. Industrial research seeks to create knowledge that has strategic advantage, protects existing business, interests or creates new opportunities [10]. At its core the dual production function of university research is the production of new knowledge that will expand the pool of knowledge and the production of new scientists. Acting as agency for each other, researchers engage in "discovery collaborations" that are mutually beneficial, permitting each to do better and go farther than if they were working alone [11]. The role of intermediary organizations, who help develop collaboration, has increased and now draws specific attention in the literature on knowledge and technology transfer [12].

This paper revisits the literature of platforms and shows how University-Industry (UI) liaisons can be studied considering the emerging and insightful literature on platforms. The aim of this study is to take an in-depth overview of the platform literature and apply the gained perspective into a successful example of UI case study. The study attempts to define the platform characteristics and contribute to the discourse by introducing UI programs as platforms. Therefore, the study contributes to research in the field by 1) developing a theoretical ground to revisit platform literature as a perspective to University-Industry programs 2) Analyzing a successful UI case study to empirically investigate the platform characteristics.

This paper is structured as follows: first, we layout the concept of platform and its characteristics and distinguish it from similar concepts such as ecosystem. Second, we discuss the University-Industry liaison literature and clarify how concepts from platform literature apply to the study of UI programs. Third, we review the case of an UI affiliate program in order to exemplify several platform characteristics. Finally, we empirically practice social network analysis and visualization to deepen our understanding of the collaborative network associated with the UI case study. The discussion and conclusion sections of the paper recap the observed characteristics of platforms within 
the case study and suggest opportunities for further study.

\section{Platforms and Ecosystems}

Ecosystems and platforms are both concepts that address interdependence across organizations and activities. The ecosystem construct is embedded in a biological metaphor that highlights multilateral relationships, rather than leaders, governance or management. Ecosystems and platforms may both have a "focal actor," who appears central to the dynamics of interaction. The "focal actor" in a platform usually plays a leadership role, benefiting from network effects and operating intentionally, often in a management or organizational capacity, in order to maximize these benefits [1], [13].

Both platforms and ecosystems are often associated with "network effects" - that is, as the network grows and more users join, the more valuable the platform becomes to the owner and to the users themselves, due to increased access to a growing network of users and complementary innovations [14]. As platforms bring together multiple user groups, they create network effects in the context of network externalities. "Increasing adoption levels can trigger positive feedback cycles that further increase the usefulness of the technology." [1]. Adner in 2017 agrees, adding that growing the relevant sides of the market in order to increase value through direct and indirect network influences is a key strategic priority in platforms and multi-sided markets [13].

Adner sees platforms as elements of a broader ecosystem, in which ecosystems are "communities of associated actors defined by their networks and platform affiliations." While ecosystems focus on structure and interdependence, platforms are concerned with governance. Platforms, he explains, "hold a hub position in a network of interactions" and "exercise power through centrality". As central actors, platforms also have an interest in the governance of interfaces, as well as their access, incentives, and control. Platforms can play the role of what Adner refers to as a "focal actor" in an ecosystem. "By increasing the number and intensity of participants in its ecosystem, the focal actor increases its bargaining power, increases system value through direct and indirect network externalities, and increases the likelihood of serendipitous interactions between partners that may unlock new interactions and combinations that increase the overall value creation of the system" [13].

Similarly, Gawer argues that platforms often have a leader or a "keystone firm" which plays a central, orchestrating role within the platform's network or ecosystem [6]. Such platform leaders manage the ecosystem to their benefit. They work to leverage network effects, "incentivizing a potentially limitless number of innovative yet autonomous agents to act in ways that are platform-enhancing, as opposed to platform-indifferent or even possibly platformcompeting." This type of governance promotes platform innovation and performance [6]. At the same time, as Gawer in 2014 explains, this approach "does not impose a priori a fixed organizational form, recognizing the multiplicity of organizational contexts within which platforms can be found." [6].

The discussion of Gawer 2014 benefits the management research agenda by introducing an organizational perspective into platform literature, which previously was defined from one of two perspectives: either a two-sided market economy [15] or a modular technological architecture [6].

This paper also adopts Gawer's Organizational approach to understanding platforms. This approach combines a specification of structure (stable core \& variable periphery) with a focus on agency [1], [6]. The Organizational approach proposed by Gawer views platforms as "evolving organizations" or "metaorganizations" that have 3 central characteristics:

- They have a modular technological architecture composed of a core and a periphery;

- They coordinate (and federate) constitutive agents who can innovate and compete;

- They create value by generating and harnessing network effects - economies of scope in supply and innovation (for the engineering design view), or economies of scope in demand (for the economics view)

In this study, we introduce a fresh perspective on University-Industry liaisons using a platform perspective to describe the role of UI programs in orchestrating and facilitating university-based multidisciplinary research that supports UniversityIndustry (UI) interactions. We approach this by utilizing data from mediaX at Stanford University, a universityindustry liaison program of the Human Sciences and Information Technologies Institute, administered in the Graduate School of Education, and serving a mission to catalyze discovery collaborations inside the university as well as create and sustain dialogues between university and industry researchers. Reviewing the evolution of research projects catalyzed by mediaX at Stanford University over two decades, we observe efforts of the platform leadership through the orchestration and evolution of collaboration networks among academic researchers. 


\section{University-Industry Liaison}

University-Industry relations broadly consist of interactions between the higher educational system and industry. When they occur, these collaborations are largely seen as an approach to improve innovation by facilitating the flow and to stimulate utilization of knowledge and experience across sectors [16]. In the knowledge industry, insights of strategic value can be increased through interdisciplinary activities, and in history of academia, interaction with industry has been a tool or vehicle to inspire business application. For academic faculty, these might include the awareness of challenging research questions with real-world applications, the opportunity to address such questions, to see their research results have tangible impacts, and to gain access to new skills, data or equipment. In parallel, companies can potentially improve business performance, for example, through inspirations to develop new techniques or technologies and by extending the capabilities and expertise available to them [17]. Thus, IU collaboration can add value to respective research efforts and can result in two-way knowledge flows between universities and industry through academic publications, conference presentations, faculty consulting, and the movement of personnel between industry and academia, to name but a few channels [18]. Science parks, incubators, interdisciplinary and multidisciplinary research hubs, and spin-off companies are also examples of results stemming from or contributing to innovative universityindustry collaborations in a knowledge-based society [19].

University-industry collaboration is particularly prevalent in US Higher Education institutions. This is due, in part, to certain structural characteristics of the system, including a lack of strong central governmental controls of policy and administration, as well as interinstitutional competition for resources and prestige, both of which create strong incentives for faculty and university administrators to develop links with industrial research. Over the course of the past century, these close research links between US universities and industry have not only produced important industrial innovations in fields ranging from pharmaceuticals to mining to agriculture, but have also influenced and fostered the development of new interdisciplinary areas of engineering and scientific research [18]. As such, university-industry collaboration is an important component of the current and future innovation ecosystem and economy [17], [20].

Successful cooperation between industry and university requires a certain synergy; both parties need to be aware of each other's interests and objectives, as well as complementary strengths [19]. Both parties must voluntarily act as agents for the interests of the other [11]. Stanford University has developed a wide range of Affiliate Programs in order to foster and catalyze industry-university relations across institutional boundaries. Industrial Affiliates Programs provide a mechanism for faculty and companies to discuss and explore broad research topics in a pre-competitive environment. Through these programs, Stanford faculty and students can learn about industry perspectives and priorities, and industry members can be exposed to new ideas and research directions. Supported by corporate membership fees, which vary by program, Industrial Affiliate Programs provide consistent emphasis on close, effective communication between stakeholders, since strong, trusting relationships between people in business and academia form the foundation for successful collaboration [17]. Rather than promoting specific technology, Industrial Affiliate Programs operate according to principles that include broad sharing of results and faculty freedom to pursue research topics of their choice. In this context, knowledge transfer often begins with industry and university interaction, and Silicon Valley is a prime example of how this resulting technology transfer has mutually benefited Industry and Higher Education [19].

\section{Case Study: mediaX as both UI Liaison and Platform Leader}

mediaX at Stanford University (mediaX) is one of the early Industry Affiliate Programs at Stanford University. mediaX is an Affiliate Program of the HSTAR Institute (Human-Sciences and Technologies Advanced Research) in the Graduate School of Education. It is a programmatic platform to support multi-disciplinary discovery relationships with initiatives that explore how understanding people can improve the design of technologies - in the areas of learning, mobility, collaboration, entertainment and commerce.

Since its inception in 2001, mediaX and its community of enterprise members and partners have funded over 100 research projects that pursue new insights on how the innovative use of communication technologies can improve the human experience.

As a multi-disciplinary vehicle (platform), the mediaX footprint reaches across the entire Stanford University community, creating a network of researchers and labs in which research questions include both human sciences and information technologies. mediaX initiatives seed campus-wide research and promote resulting insights for industry interests. With small grants awarded for concept-proving research on Research Themes of interest to mediaX member 
organizations, researchers conduct studies that test the relevance of academic concepts for real world problems and experiment with concept proofs that expand research methods. Through dialogue and collaboration, university and industry researchers discuss the questions, research findings and resulting insights; together they challenge current knowledge and stretch intellectual resources to gain new insights relevant to both industry and academic realms.

mediaX Research Themes articulate questions that have a time horizon of three to seven years and often revolve around complex issues that are not yet well defined. Fielded through requests for proposals across all collegiate units at Stanford University, professors and researchers affiliated with labs from multiple disciplines, departments and collegiate units propose innovative research approaches to the challenge articulate in the Research Theme. Seen from the perspective of industry members, the Research Theme process identifies novel research pathways and new approaches to pursuing critical issues; it lowers the risk of exploration for industry. mediaX Research Themes allow the industry members to leverage the Stanford network to enhance their existing expertise, identify needs for new expertise, and engage with current research methods and results, stimulating new insights on company questions. Through Research Themes, participating researchers from the Colleges of Engineering, Humanities and Sciences, as well as the Graduate School of Education, Schools of Law, Medicine, and Business have identified and pursued critical questions to discover new insights on how information technology affects people's lives, how to better design products and services to make them more usable.

The context of mediaX includes Silicon Valley's ecosystem of established companies, startups, high quality research institutions and varied educational programs, along with an entrepreneurial culture, actively engaged industry partners, Stanford thought leadership, and the energetic creativity of bright motivated graduate students and post-doctoral students. This external ecosystem infuses the mediaX Research Theme program with unique externalities that mediaX as the platform leader uses to activate the full range of technological, cultural and intellectual resources at Stanford University.

A key component to keeping all of the collaborative parts well lubricated is an open and efficient schedule of dialogue and communications. Highlighting, recapping and promoting the findings of research projects is critical to information flows. Through a variety of communication mechanisms (video, audio, graphic illustrations, reports, white papers and photos), mediaX promotes the research and resulting insights. These findings and results are then made public using mediaX's multi-platform distribution networks, again targeting the specific audiences that need to be reached. With precise targeting of messages, mediaX is able to remove many roadblocks that encumber the collaboration efforts of University researchers and Industry researchers, thereby enhancing the network effects and platform value to all participants.

\section{Empirical: Network Analysis, Data Curation \& Visuals}

In this case study, the collaborative network has been catalyzed and orchestrated through efforts of the platform leader. The formation and development of a network of participants has been facilitated by funding multidisciplinary academic research proposals that respond to challenging themes that reflect the concerns of industry. Collaborations of industry and university researchers are stimulated by shared interest in novel research questions. Collaborations among academic colleagues in diverse departments and collegiate units are stimulated by competitive opportunities to define and test new conceptual approaches to those research questions.

The development of the collaboration network among participants in academic projects can be revealed using social network analysis (SNA). SNA is the process of investigating social structures using networks and graph theory [21]. It characterizes networked structures in terms of nodes (individual actors, people, or things within the network) and the ties, edges, or links (relationships or interactions) that connect them [22]. SNA is used widely in the social and behavioral sciences, as well as in economics, marketing, and industrial engineering [23], [24]. The snapshot-type visualization of patterns and structures of the network enables a qualitative narrative for this case study. In this case, we view the unstructured collaborative network of projects through the lens of SNA in order to investigate collaborative network evolution.

Figure 1 was created from 216 individual researchers (nodes) who participated in mediaX research projects over a period of 15 years, in which 18 Research Themes were fielded. The color of the nodes represents the participant's collegiate affiliation at Stanford. External affiliates include academics from universities outside of Stanford, as well as industry researchers.

In Figure 1, the College of Engineering (CoE) represents $32.87 \%$ of the participating researchers, Humanities and Sciences (H\&S) represent 21.3\%, Graduate School of Education (GSE) makes up 14.35\%, School of Medicine makes up $6.48 \%$, School of Law 
makes up 2.78\%, and External affiliates are $10.19 \%$.

Figure 2 illustrates the intercollegiate collaborations of the 216 researchers (Nodes) on 110 projects under 18 themes, generating 491 collaboration events (Edges) among the six major schools and external affiliates. The edges in the network reflect collaborations initiated through funded projects which provided collaboration occasions between the individuals participating in those projects.

Multiple edges in Figure 2 between researchers in the College of Engineering and the College of Humanities \& Sciences reveal multidisciplinary responses to the Research Themes. Collaborations between the external affiliates and researchers in the Graduate School of Education and the College of Engineering indicate shared interests that were activated by the Research Themes. Edges represent collaborations that occurred for the purpose of the project work between individuals.

Overtime, strategic alignment and introduction of projects by the platform leader have resulted in the engagement within research projects by participants from various schools. Figure 3 illustrates the trend over time of researchers' participation, collaboration activity and whether that collaboration was multidisciplinary, as shown by inter-collegiate interactions. While the data show an overall tendency for collaboration to occur among researchers within the schools, it also shows that the portion of multidisciplinarity interaction is higher for some of the Research Themes.

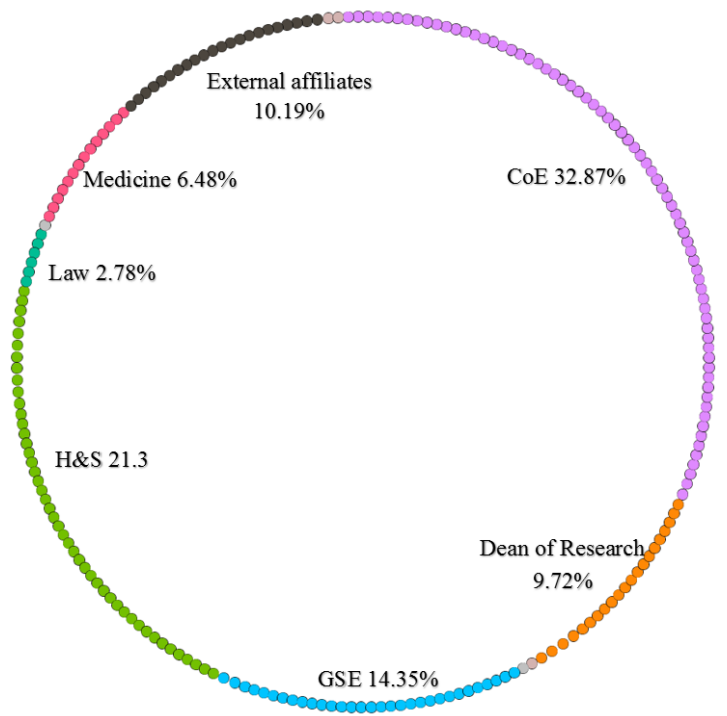

Figure 1. Collegiate Units of Researchers.

Nodes: 216 individual researchers, including faculty members, postdocs, graduate students, visiting scholars and industry researchers

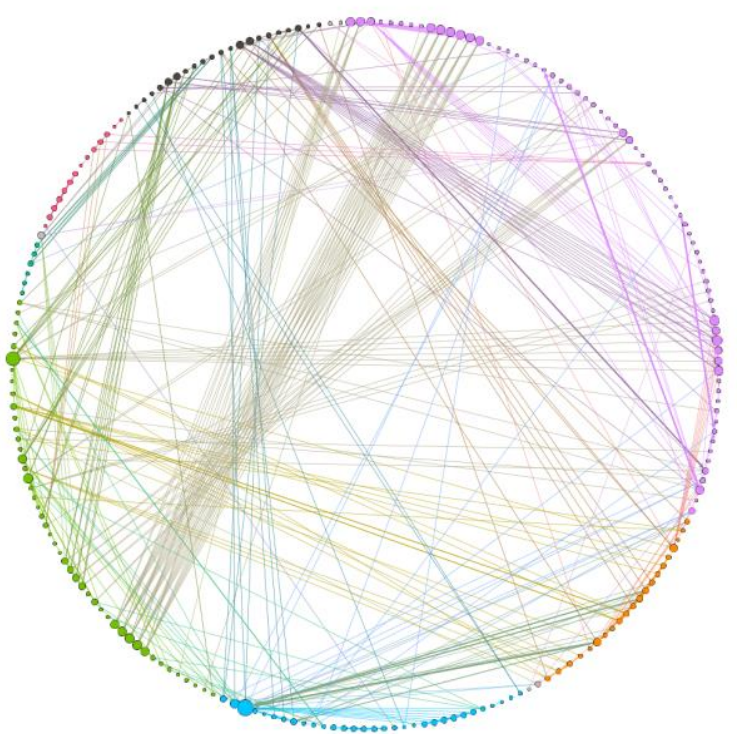

Figure 2. Intercollegiate Collaborations Among Researchers. Nodes represent individuals, Colors represent colleges/schools, Edges represent collaboration between nodes.

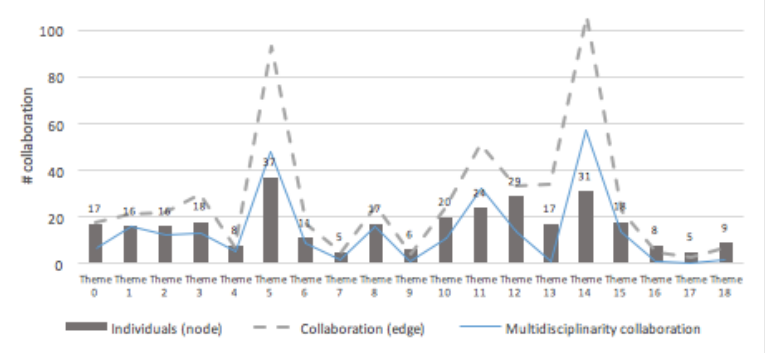

Figure 3: Researchers' participation, collaboration and multidisciplinary collaboration over project themes

In Figure, 3 bar height signifies number of researchers participating in projects awarded for a specific Research Theme. Dotted lines represent collaborations on projects within the Themes. The solid lines indicate the multidisciplinary nature of collaborations, counted as multidisciplinary when collaborations occur between two individuals from different collegiate units.

Over the course of this case study, several Research Themes resulted in greater increases in participation and collaboration relationships. Responses to Requests for Proposals are naturally dependent on many factors, including availability of other sources of funding, interested graduated students, and faculty time. Additionally, the topic of each Research Themes is a primary mechanism for orchestrating collaborations. The Research Themes included in this case study show 
that three topics sparked higher levels of response and collaboration, as indicated in Table 1.

Table 1. Multidisciplinary Impact of Research Themes. (Themes with high collaboration impact are shown in bold.)

\begin{tabular}{c|l} 
Theme & Topic \\
\hline 0 & Social and Computing Sciences \\
1 & Sensing and Control \\
2 & Learning and Training \\
3 & Video Processing and, Cataloging \\
4 & Sensing and Control \\
6 & Social Interaction and Collaboration \\
7 & Mobile Devices in Collaboration \\
8 & Onotion Detection Facial Expressions \\
9 & Mobile Devices \\
10 & Human Machine Interaction and Sensing \\
11 & Virtual and Physical Realities \\
12 & Productivity of Knowledge Workers \\
13 & Future of Content \\
14 & Publish on Demand \\
15 & Insights from Digital Learning \\
16 & Memory, Estates and Legacies \\
17 & Future Contexts for Smart Mobile \\
18 & Devices \\
\end{tabular}

The Requests for Proposal for Themes 5, 11 and 14 specifically requested multidisciplinary and collaborative proposals. Theme 11 was introduced following a workshop and conference promoting the area of inquiry, as well as the range of potential questions for scholarly inquiry and business application.

The network dynamics of the multidisciplinary ecosystem of mediaX-funded research projects also reveal insights about the platform leader's role in catalyzing and orchestrating collaborations among university researchers. In Figures 4 through 12, shown at the end of the paper, each collaboration between researchers is shown with an edge. Lighter edges indicate single project collaborations. Darker edges indicate repeated collaborations on projects between the same researchers. In these visualizations, clusters develop over time as projects by new researchers are funded, some of whom are collaborating.

As shown in Figure 4, which includes data from Themes 0 to 2, the first three Research Themes make 17 collaborative edges which equal3.76\% visibility of the edges in the full network. Multidisciplinary participation is evidenced, several joint projects are revealed, and two team clusters appear. In Figure 5 (including data from Themes 3 \& 4), additional researchers from multiple collegiate units are added, new collaboration clusters appear, and a repeated collaboration (shown in the dark link between nodes in two different clusters) bridges two clusters of the network.

Through Theme 5, shown in Figure 6, additional researchers are added to the mediaX ecosystem, increasing the size and diversity of the ecosystem and adding and expanding the clusters in the network. Additional nodes and edges are added in subsequent Themes, shown in Figures 7 through 12, growing the ecosystem to the 216 nodes and 491 edges revealed in the cumulative network of Figure 2.

Over the course of 18 Research Themes, the cumulative collaboration network shows five distinct multidisciplinary clusters larger than five participants. Eight small multidisciplinary clusters with 3 to 5 collaborators are also visible. Bridges between small clusters evolve with repeated collaborations into larger clusters.

Figure 13 shows the last evolutionary phase of the network (including Themes 17-18), in which the single and repeated collaborations between nodes evolve to large clusters of multidisciplinary collaborations. The node colors reveal that Cluster 1 includes a heavy proportion of researchers from the Graduate School of Education; Cluster 2 includes a heavy proportion of researchers from the College of Engineering. A collaboration between two researchers in the College of Humanities and Sciences bridges these two large clusters. In social networks, network bridges can be interpreted as ties from one group to another group, enabling the transmission of certain values in the network [25], as well as resources such as talent, information, and material assets [26].

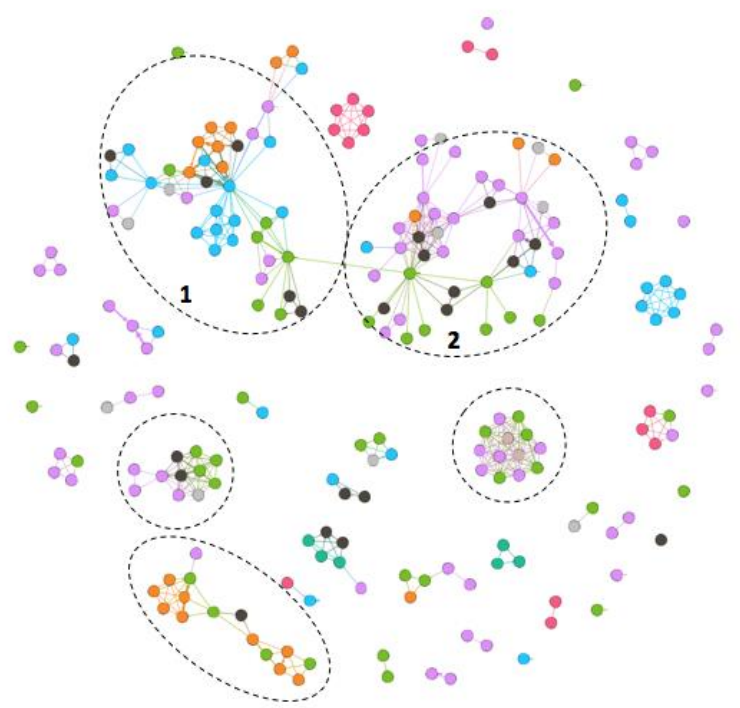

Figure 13. Enlargement of the last evolutionary segment of collaborative network, including themes 1718. Clusters highlighted with dotted lines by authors. 
The collaborative network illustrated in Figure 13 indicates properties such as weak ties and strong ties developed by Granovetter in 1983 [25]. The heavier edges within small groups show a co-occurrence of collaborations among small groups of individuals. The cumulative network of collaborations also includes occasional collaborations - weaker links - both inside and outside of the clusters. In mathematical sociology, interpersonal ties are defined as information-carrying connections between people. Generally speaking, interpersonal ties come in three varieties: strong, weak or absent. Weak ties, it is argued, are responsible for the majority of the embeddedness and structure of social networks - in society as well as the transmission of information through these relational networks [25].

Specifically, more novel information flows to individuals through weak rather than strong ties. Because our close friends tend to move in the same circles that we do, the information they receive overlaps considerably with what we already know. Acquaintances, by contrast, know information and other people that we do not know, and thus have the potential to exchange more novel information [27].

\section{Analysis and Discussion}

Network visualizations showing the evolution of the mediaX research ecosystem at Stanford through the course of 18 Research Themes illustrate both stable and transitory participation in research topics at the intersection of human sciences and information technologies. As Granovetter's theory of strong and weak ties suggests, the core group of researchers reveals repeated collaborations over time; these are the strong ties of the network. The network's weaker, more transitory ties provide ever changing input to the changing topics of mediaX Research Themes and enrich the ecosystem with diverse perspectives.

The context of mediaX's platform operation includes the key influences of interdisciplinary research in academic environments - personal, disciplinary and administrative dimensions [28]. Participating researchers include faculty members across departments in 6 collegiate units, at a variety of academic levels graduate students, post docs, professional research staff and external affiliates, both academic and industrial. At the personal level, relationships among faculty members provide mechanisms for graduate students from multiple departments to work together on innovative concepts that extend beyond single disciplines. Research funding for projects facilitates this collaboration sometimes formally, sometimes informally. Additionally, Stanford administrative policies permit funds to be transferred among departments and across colleges, reducing the barriers to collaboration by permitting material resources to flow alongside talent and information [28]. The evolution of the collaboration clusters throughout the time and over various Research Themes of this case study illustrates the potential for autocatalytic connections across multiple layers of the ecosystem [29].

Within the university-industry context, the many affiliate programs at Stanford University reveal wide variety in the attributes of UI affiliate programs as platforms. mediaX implements its platform leadership role as a research catalyst. An open platform by Gawer's definition in 2014, the platform leadership of mediaX is exercised through the creation of Research Themes, reflecting interests of industry members, and through research grants, and through the exploration of these Themes through Stanford research projects, connecting a multidisciplinary and inter-collegiate ecosystem of researchers who elect to explore questions pertinent to the Research Themes.

The mediaX platform connects researchers at Stanford and adds value by creating a shared vision of the discovery challenge embedded in a Research Theme. Through discovery challenges in the Research Themes and discovery goals in the awarded projects, faculty members - their respective research teams, labs, centers, and institutes - are loosely coupled [30] with each other and with the industry affiliates whose interests are leveraged through their memberships, the research challenge and their participation in dialogues. New links in the network are created through new research awards on new Research Themes.

The mediaX platform connects industry and university researchers. The Research Themes themselves are inspired by issues of interest to one or more of the affiliated industry member organizations, sculpted appropriately for academic inquiry, linking the academic research to critical issues external to Stanford University. Continual dialogue and communication activities promote the exchange of questions, practices, information, and insights. These interactions build relationships that often last beyond the research projects themselves.

Strong ties are celebrated and loose ties are nurtured through a combination of technology transfer mechanisms [31], including formal and informal conversations and discussions among project researchers and between industry liaisons and Stanford researchers, conferences, workshops, arrangements for visiting scholars, and publications.

Gawer's Organizational framework of platforms lays a theoretical foundation for platform research. This paper uses the Organizational framework of platforms to describe a case study of one externally-oriented and 
multidisciplinary platform, situated in a university. In this manner, the paper departs from traditional uses of platform concepts as internal, company-specific platforms or digital platforms [14]. Externally-oriented, industry-wide platforms can be focused on products, technologies or even services. Their role is to "provide the foundation upon which external innovators, organized as an innovative business ecosystem, can develop their own complementary products, technologies, or services" [14].

In this case study, we reveal how a platform leverages inspiration and resources from external innovators to create a multidisciplinary ecosystem, composed of a core and a periphery. The platform coordinates (and enables the federation) of researchers (constitutive agents) who compete for research grants and innovate through novel research. The platform creates value by generating and harnessing the network effects of talent, information and financial resources.

As scholars and as practitioners, we are still in the early stages of understanding the roles that platforms will play in shaping future collaboration and competition. Yet, the value of an ecosystem as backup to a platform is already understood as a long term strategy that extends the value of having an excellent initial offering [32].

\section{Practical Implication}

This study showed the importance of the leadership in the platform for the evolution of the collaboration clusters throughout the time and over various research themes. The platform leadership role as a research catalyst in the context of university industry liaison can be exercised through the creation of research themes, reflecting interests of industry members, and through research grants. Facilitation from administration stand point in funds to flow alongside talent and information has been found quite essential as well.

The major role of the UI liaison is to bridge the gap between university and industry and this can be done by reflecting the affiliated industry member's interest for academic inquiry. Furthermore, exploration of important themes through research projects going on in the research institute would be a necessary effort. The act of the UI can catalyze the attempt to connect a multidisciplinary and inter-collegiate ecosystem of researchers who elect to explore questions pertinent to the research themes.

Forward-looking research agenda of the UI liaison can provide not only a progressive approach to research but also an attractive direction for various disciplinary approaches so to collaborate. Exploratory nature of the new research topic increase the tendency for formation of multidisciplinary groups which is decisive for growing of the collaborative network.

\section{References}

[1] M. de Reuver, C. Sørensen, and R. C. Basole, "The digital platform: a research agenda," J. Inf. Technol., 2017.

[2] M. DE REUVER, H. BOUWMAN, and T. HAAKER, "Business Model Roadmapping: a Practical Approach To Come From an Existing To a Desired Business Model," Int. J. Innov. Manag., vol. 17, no. 1, p. 1340006, 2013.

[3] M. De Reuver, E. Verschuur, F. Nikayin, N. Cerpa, and H. Bouwman, "Collective action for mobile payment platforms: A case study on collaboration issues between banks and telecom operators," Electron. Commer. Res. Appl., vol. 14, no. 5, pp. 331-344, 2015.

[4] F. Svahn, R. Lindgren, and L. Mathiassen, “Applying options thinking to shape generativity in digital innovation: An action research into connected cars," Proc. Annu. Hawaii Int. Conf. Syst. Sci., vol. 2015-March, pp. 4141-4150, 2015.

[5] L. L. Kiesling, "Implications of Smart Grid Innovation for Organizational Models in Electricity Distribution," Forthcoming, Wiley Handb. Smart Grid Dev., 2015.

[6] A. Gawer, "Bridging differing perspectives on technological platforms: Toward an integrative framework," Res. Policy, vol. 43, no. 7, pp. 1239-1249, 2014.

[7] M. Perkmann, V. Tartari, M. McKelvey, E. Autio, A. Brostr??m, P. D'Este, R. Fini, A. Geuna, R. Grimaldi, A. Hughes, S. Krabel, M. Kitson, P. Llerena, F. Lissoni, A. Salter, and M. Sobrero, "Academic engagement and commercialisation: A review of the literature on universityindustry relations," Res. Policy, vol. 42, no. 2, pp. 423-442, 2013.

[8] M. Dalziel and S. Parjanen, "Measuring the impact of innovation intermediaries: A case study of tekes," Pract. Innov. Insights, Appl. Policy Implic., pp. 117-132, 2012.

[9] M. Bordons, M. A. Zulueta, F. Romero, and S. Barrigón, "Measuring interdisciplinary collaboration within a university: The effects of the multidisciplinary research programme," Scientometrics, vol. 46, no. 3, pp. 383-398, 1999.

[10] H. Etzkowitz, "Innovation in innovation: the Triple Helix of university-industry-government relations," Soc. Sci. Inf. Sur Les Sci. Soc., vol. 42, pp. 293-337, 2003.

[11] M. G. Russell and K. Still, "Engines driving knowledgebased technology transfer in business incubators and their companies," Proc. 32nd Annu. Hawaii Int. Conf. Syst. Sci., p. 9, 1999. 
[12] M. Wright, B. Clarysse, A. Lockett, and M. Knockaert, "Mid-range universities' linkages with industry: Knowledge types and the role of intermediaries," Res. Policy, vol. 37, no. 8, pp. 1205-1223, 2008.

[13] R. Adner, "Ecosystem as Structure: An Actionable Construct for Strategy," J. Manage., vol. 43, no. 1, pp. 39$58,2017$.

[14] A. Gawer and M. A. Cusumano, "Industry platforms and ecosystem innovation," J. Prod. Innov. Manag., vol. 31, no. 3, pp. 417-433, 2014.

[15] J. C. Rochet and J. Tirole, "Platform competition in twosided markets," Compet. Policy Int., vol. 10, no. 2, pp. 180 218, 2014.

[16] S. Ankrah and O. AL-Tabbaa, "Universities-industry collaboration: A systematic review," Scand. J. Manag., vol. 31, no. 3, pp. 387-408, 2015.

[17] A. Dowling, "The Dowling Review of BusinessUniversity Collaborations," Bis, no. July, 2015.

[18] D. C. Mowery, J. E. Oxley, and B. S. Silverman, "Strategic alliances and interfirm knowledge transfer," Strateg. Manag. J., vol. 17, no. S2, pp. 77-91, 1996.

[19] Y. Fassin, "The strategic role of university-industry liaison offices," J. Res. Adm., 2000.

[20] H. Etzkowitz and L. Leydesdorff, "The dynamics of innovation: from National Systems and Mode 2', to a Triple Helix of university-industry-government relations," Res. Policy, vol. 29, pp. 109-123, 2000.

[21] E. Otte and R. Rousseau, "Social network analysis: a powerful strategy, also for the information sciences," pp. 441-453, 2002.

[22] M. Grandjean, "A social network analysis of Twitter: Mapping the digital humanities community,"

Http://Www.Editorialmanager.Com/Cogenthumanities, 2016.

[23] A. D'Andrea, F. Ferri, and P. Grifoni, "An Overview of Methods for Virtual Social Network Analysis," Comput. Soc. Netw. Anal. Trends, Tools Res. Adv., pp. 3-26, 2009.

[24] A. Hajikhani, J. Porras, and H. Melkas, "Brand Analysis in Social Network Services: Results from Content Analysis in Twitter Regarding the US Smartphone Market," Int. J. Innov. Technol. Manag., p. 1740008, Oct. 2016.

[25] M. Granovetter, "The Strength of Weak Ties: A Network Theory Revisited," vol. 1, no. Sociological Theory, pp. 201-233, 1983.
[26] M. G. Russell and C. Hall, "Network visualisations of knowledge assets : their value and user experiences for innovation development," vol. 16, no. 3, pp. 221-242, 2017.

[27] M. Granovetter, "The Impact of Social Structure on Economic Outcomes," J. Econ. Perspect. 19 (1 ) 33-50., 2005.

[28] M. G. Russell, Administering Interdisciplinary Collaboration. Elsevier B.V., 1985.

[29] S. E. Page, "John F. Padgett and Walter W. Powell: The Emergence of Organizations and Markets," Adm. Sci. Q., vol. 58, no. 2, pp. 304-306, Jun. 2013.

[30] K. E. Weick, "Educational Organizations as Loosely Coupled Systems Author ( s ): Karl E . Weick Source: Administrative Science Quarterly, Vol . 21 , No . 1 ( Mar ., 1976 ), pp . 1-19 Published by: Sage Publications, Inc . on behalf of the Johnson Graduate School of," vol. 21, no. 1, pp. 1-19, 2017.

[31] D. V. Gibson and E. M. Rogers, "R \&amp; D collaboration on trial : the Microelectronics and Computer Technology Corporation,” p. 607, 1994.

[32] M. Cusumano, "Technology strategy and management: The evolution of platform thinking," Commun. ACM, vol. 53, no. 1 , p. 32, 2010. 


\section{Appendix}
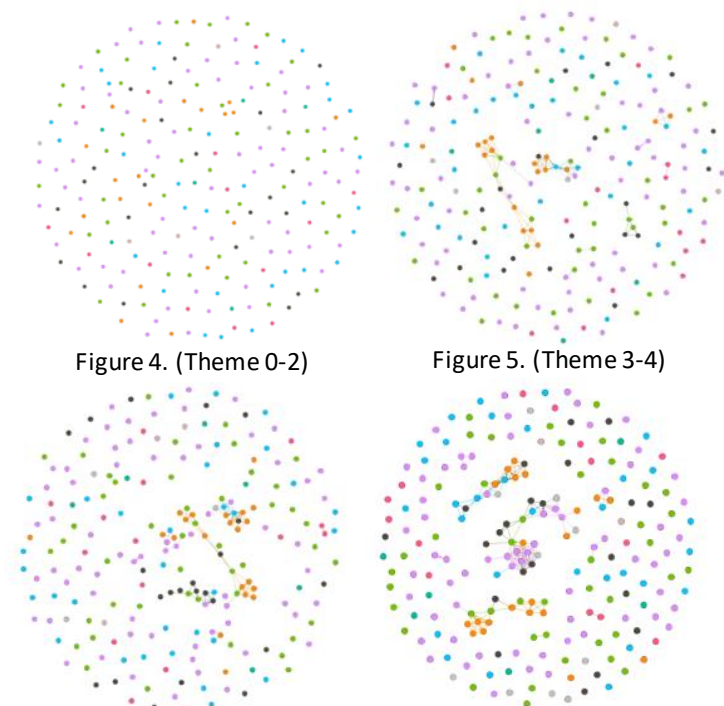

Figure 5. (Theme 3-4)

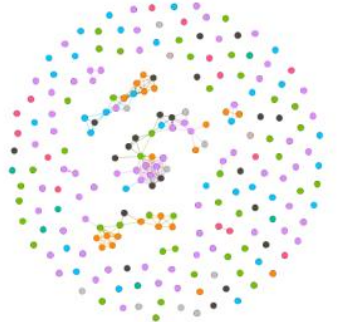

Figure 6. (Theme 5)

Figure 7. (Theme 6-7)
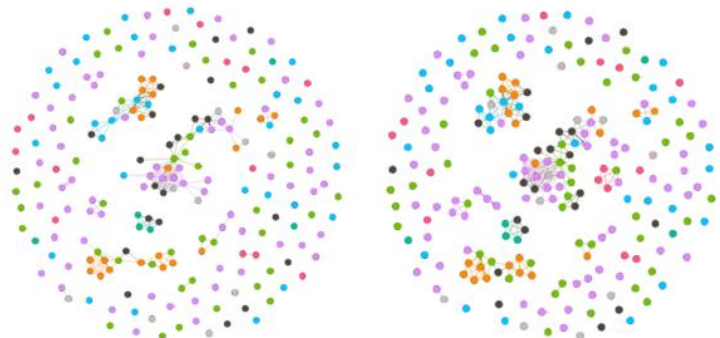

Figure 8. (Theme 8-9-10)

Figure 9. (Theme 11)

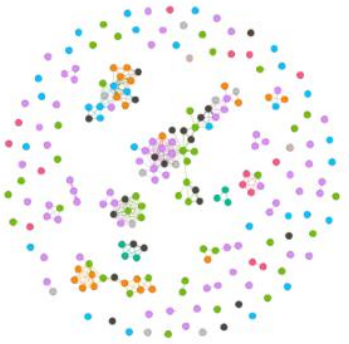

Figure 10. (Theme 12-13)

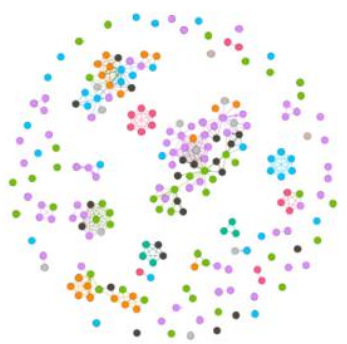

Figure 11. (Theme 14)

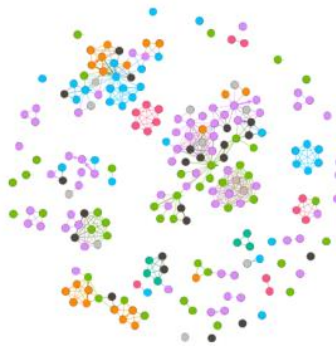

Figure 12. (Theme 15-16)

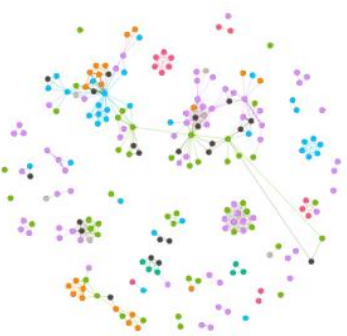

Figure 13. (Theme 17-18)

Figures 4 through 13. Development of Multidisciplinary Network and Collaboration Clusters Through Research Themes. Nodes represents individuals, Colors represent collegiate units, Edges represents collaboration between nodes. 